\title{
LOW BACK PAIN IN BETA THALASSEMIA MAJOR REVEALING SACRAL EXTRA MEDULLAY HEMATOPOEISIS: A CASE REPORT
}

Rita Ahmad ${ }^{1}$, Lina Okar ${ }^{2}$, Hussam Almasri², Mouhammad Sharaf Eldean ${ }^{2}$, Abdelhaleem Elhiday $^{2}$, Fateen Ata ${ }^{2,2}$, Amna Gamil ${ }^{2}$, Ali Barah ${ }^{2}$, Elsayed, Ahmad Mounir ${ }^{2}$, and mohamed yassin ${ }^{3}$

${ }^{1}$ Affiliation not available

${ }^{2}$ Hamad Medical Corporation

${ }^{3} \mathrm{HMC}$ NCCCR

September 28, 2020

\begin{abstract}
Extramedullary hematopoiesis (EMH) represents the production of blood cells outside of the bone marrow and occurs in beta thalassemia major. Although EMH usually occurs in the liver, spleen, and lymph nodes, it may also occur in the spinal canal or sacral bone lead to neurological complications
\end{abstract}

\section{INTRODUCTION}

Beta thalassemia major is the severe form of beta thalassemia, which is caused by mutations in beta globin gene, either reduced $\left(\beta^{+}\right)$or absent $\left(\beta^{0}\right)$ in hemoglobin A resulting in unbound $\alpha$ globin chains that accumulate in erythroid precursors in the bone marrow and in the mature erythrocytes leading to ineffective erythropoiesis and peripheral hemolysis. About $1.5 \%$ of the world population are carriers of $\beta$ thalassemia [1].

In severe disease, extramedullary hematopoiesis (EMH) -which is production of blood elements outside the bone marrow- occurs mostly in the liver and the spleen but in rare cases can manifest as bony masses that behave clinically like tumors, leading to optic nerve atrophy, spinal cord compression and other clinical scenarios $[2,3]$.

Here we present a case of a 31-year old Pakistani woman with transfusion-dependent beta thalassemia (TDBT), who complained of low back pain due to a mass reflecting extra medullary hematopoiesis which very rarely occurs in the sacrum as there was only one case reported in the literature and this is the second one.

\section{CASE PRESENTATION}

A 31-year-old Pakistani female presented with generalized body pain and low back pain. Her past medical history was remarkable for transfusion-dependent beta thalassemia (TDBT) major since childhood complicated with iron overload as per her cardia and hepatic MRI reports, receiving iron chelation therapy (Deferasirox $1080 \mathrm{mg}$ daily), type II diabetes mellitus on Insulin therapy and migraine. her past surgical history includes splenectomy on 2012. She has a long history of body pain. She was recently admitted due to COVID-19 infection and discharged from quarantine facility one week earlier after a smooth course of infection. 
She presented to the emergency department complaining of body pain for the last few days and dysuria. She has similar complains on and off for many years, yet the pain increased recently, and it is mainly in the hip and lower abdomen area (suprapubic), the pain increases with urination, she has also back pain for the last three months more severe at night, responds partially to analgesia, radiates to the hip and is limiting her movement. On admission vitals were:

BP 107/67 mmHg,

HR $95 / \mathrm{min}$

Temperature $36.7 \mathrm{o}$

Saturation $96 \%$ on room air.

Primary investigations included: Labs, MRI pelvis and hips, Abdomen US showed the following:

Labs: On admission:

Her labs revealed hypothyroidism, so she was started on Levothyroxine therapy.

$\mathrm{X}$ ray pelvis and left hip:

Showed decreased bone density, bilateral coxa profunda.

US abdomen showed mildly enlarged fatty liver $(18.2 \mathrm{~cm})$.

US pelvic: Normal study.

MRI Hips and pelvic: Diffuse bone marrow reconversion and Presacral soft tissue mass as described, most likely extramedullary hematopoiesis.

So as per MRI the patient has extramedullary hematopoiesis presenting as soft tissue mass in the presacral area, from surgical point of view, the mass is not accessible, but still need to be excised. After that cervical and lumbar MRI spine was done to look if there are other extramodular hematopoietic sites and it showed:

Diffusely decreased T1 marrow signal intensity of the entire visualized bones which is in keeping with red marrow reconversion. Redemonstrations of a presacral well defined lobulated soft tissue mass measuring $5.3 \times 3 \mathrm{~cm}$, it is separated from the underlying sacrum, no sacral destruction. The mass is showing mild postcontrast enhancement suggestive of extramedullary hematopoiesis. Another similar smaller extradural mass measuring $2 \mathrm{~cm}$ is seen posterior to $\mathrm{S} 1$ vertebral body at the midline laterally displacing $\mathrm{S} 1$ traversing nerve roots.

Currently: she is still on blood transfusion to keep HB more than 10 in case of any surgical procedure was decided to be done, plan now is toward the excision of the mass. The patient is taking the following medications: Celecoxib $200 \mathrm{mg}$ PRN, deferasirox $1080 \mathrm{mg}$ oral daily, insulin therapy and vitamin D supplementation, Levothyroxine $75 \mathrm{mg}$ daily, tramadol $50 \mathrm{mg}$ oral BID.

\section{DISCUSSION}

Thalassemia is the most common hemoglobinopathy, almost $5 \%$ worldwide have at least one thalassemia defect allele. It is highly prevalent in Southeast Asia, the Asian-Indian subcontinent, and the Mediterranean region $[1,4]$.

It is categorized into major two groups, $\alpha$ thalassemia and $\beta$ thalassemia.

$\alpha$ thalassemia is caused by deletions in one or more of the four alpha globin genes. Deletion of two alpha genes causes thalassemia alpha minor $(-, \alpha \alpha)$ or $(-\alpha,-\alpha)$, while loss of three genes results in hemoglobin $\mathrm{H}$ disease $(-,-\alpha)$, and deletion of all four genes leads to hemoglobin Barts or hydrops fetalis.

$\beta$ thalassemia on the other hand, results from mutations rather than deletions in beta globin genes ranging from reduced production of beta chains $\left(\beta^{+}\right)$and asymptomatic disease as in beta thalassemia trait or minor 
to absence of beta chains causing severe course of the disease seen in beta thalassemia major $\left(\beta^{0}\right)[4]$. The absence of beta globin chains results in excess unbound $\alpha$ globin chains that accumulate and precipitate in erythroid precursors in the bone marrow and in the mature erythrocytes, leading to ineffective erythropoiesis and peripheral hemolysis [1].

TDBT or also known as Cooley's anemia, refers to the most severe form of the disease where there is minimal to no beta globin chain production and as a result little to absent $\mathrm{HbA}$.

Patients with beta thalassemia major have profound and lifelong transfusion-dependent anemia, which affects their quality of life and performance status, especially among children patients as it largely affects their academic performance as well as their emotional and psychological status [5]. Symptoms typically begin to manifest relatively late after birth (6 to 12 months of age) because major hemoglobin in newborns is fetal hemoglobin $(\mathrm{HbF})$, which consists of gamma chains instead of beta chains. Symptoms include anemia which is severe and profound and if untreated can be as low as $3-4 \mathrm{mg} / \mathrm{dl}$, it's typically microcytic hypochromic with elevated RBC count. Gallstones formation and jaundice due to chromic hemolysis which also leads to hepatosplenomegaly which can be exacerbated by extramedullary hematopoiesis. Patients who develop cytopenias and shortened survival of transfused RBCs may be evaluated for splenectomy [6]. TDBT as the name implies carries the risks associated with recurrent blood transfusions, especially iron overload that requires monitoring and management by long-term iron chelation therapy to prevent iron-overload complications such as cardiomyopathy and endocrinopathy, which carry high morbidity and mortality rates [7]. However, iron overload is a one complication that paves the way for subsequent serious complications. Osteoporosis is recognized due to different etiologies including chronic anemia, ineffective hematopoiesis and bone marrow expansion, calcium and zinc deficiencies. But the exact mechanism is still not fully understood [8]. Other complications include thyroid disease particularly hypothyroidism, primary rather than secondary [9], as seen in our patient with TSH of $20 \mathrm{mIU} / \mathrm{L}$ with no other signs or symptoms suggesting secondary cause, and was started on levothyroxine during admission. Excess iron also leads to diabetes and glucose abnormalities, hypogonadotropic hypogonadism due to chronic liver disease and impaired pituitary axis which also can cause growth hormone deficiency $[10,11,12]$.

Extramedullary hematopoiesis is the production of blood cells outside the bone marrow that typically occurs in liver and spleen and leads to hepatosplenomegaly, although rarely can result in skeletal malformations such as facial deformities and change in body habitus. It also may manifest in the mediastinum and produce symptoms as cough or shortness of breath mimicking thoracic tumors [2]. However, spinal cord compression (SCC) is a rare manifestation of EMH although it may be asymptomatic, EMH-associated SCC can lead to permanent neurological injury, and when that occurs, surgical decompression should be considered with or without radiotherapy, although surgery is not always possible and may carry potential complications [13].

MRI is the diagnostic modality of choice, and biopsy is not always needed to confirm the diagnosis, but when performed it can be CT-guided as was done in our case.[13].

In our patient the main symptom was low back and low abdominal pain, without neurological symptoms. The MRI showed soft tissue mass in the presacral area representing EMH. Patient was started on hydroxyurea and hypertransfusion. Treatment options for EMH at this site is either radiotherapy or surgical excision. Because of the patient's young age and the risk of infertility, surgical excision was preferred to radiotherapy particularly as the lesion is also well-localized and operable.

The following images show MRI lesions and biopsy results.

\section{CONCLUSION}

EMH is a well-established complication of thalassemia major and can manifest in variable forms ranging from asymptomatic hepatosplenomegaly to skeletal malformations causing serious adverse effects such as SCC which is rarely encountered but should always be held in mind when assessing patients with TDBT.

\section{CONFLICT OF INTEREST}


All authors have declared that they do not have conflict of interest.

\section{AUTHOR CONTRIBUTION}

Rita Ahmad, MD: first author, manuscript writing, literature review

Lina Okar, MD: case-presentaion writing

Hussam Almasri, MD: manuscript writing

Fateen ata, MD: literature review

Abdelhaleem Elhiday, MD: Literature review

Amna Gamil, MD: Literature review

Ali Barah, MD: providing radiological images

M. Z. Sharaf Eldean, MD, FCAP: histopathlogic diagnosis and slides

Elsayed,Ahmed Mounir, MD: surgical point of view and literature review

Mohamed A Yassin, MBBS, MSc: Mentorship, manuscript writing, literature review

\section{REFERENCES}

1. De Sanctis V, Kattamis C, Canatan D, Soliman AT, Elsedfy H, Karimi M, Daar S, Wali Y, Yassin M, Soliman N, Sobti P. $\beta$-thalassemia distribution in the old world: an ancient disease seen from a historical standpoint. Mediterranean journal of hematology and infectious diseases. 2017;9(1).

2. Abdulla MA, Yassin MA, Abdelrazek M, Mudawi D, Ibrahim F, Soliman DS, ElOmri H, Nashwan AJ, Fernihough L, De Sanctis V, Soliman AT. A persistent cough as atypical clinical presentation of intrathoracic extramedullary hematopoiesis $(\mathrm{EMH})$ in a female with thalassemia intermedia. Acta Bio Medica: Atenei Parmensis. 2018;89(Suppl 2):41.

3. Garg K, Singh PK, Singh M, Chandra PS, Sharma BS. Long segment spinal epidural extramedullary hematopoiesis. Surg Neurol Int . 2013; 4:161. Published 2013 Dec 26. doi:10.4103/2152-7806.123657

4. Martin A, Thompson AA. Thalassemias. Pediatr Clin North Am. 2013;60(6):1383-1391. doi:10.1016/j.pcl.2013.08.008

5. Nashwan AJ, Yassin MA, Babu GD, Nair SL, Libo-on IL, Hijazi HA, De Sanctis V, Soliman A. Quality of life among adolescents aged 14 to 18 years with beta-thalassemia major (TM) in Qatar. Acta Bio Medica: Atenei Parmensis. 2018;89(Suppl 2):16.

6. Tassiopoulos T, Rombos Y, Konstantopoulos K, Revenas K, Tassiopoulos S, Aessopos A. Spleen size in beta-thalassaemia heterozygotes. Haematologia (Budap). 1995;26(4):205-209.

7. Kanbour I, Chandra P, Soliman A, De Sanctis V, Nashwan A, Abusamaan S, Moustafa A, Yassin MA. Severe liver iron concentrations (LIC) in 24 patients with $\beta$-thalassemia major: correlations with serum ferritin, liver enzymes and endocrine complications. Mediterranean journal of hematology and infectious diseases. 2018;10(1).

8. Yassin MA, Soliman AT, De Sanctis V, Abdelrahman MO, Bedair EM, AbdelGawad M. Effects of the anti-receptor activator of nuclear factor kappa B ligand denusomab on beta thalassemia major-induced osteoporosis. Indian journal of endocrinology and metabolism. 2014 Jul;18(4):546.

9. Soliman AT, Al Yafei F, Al-Naimi L, Almarri N, Sabt A, Yassin M, De Sanctis V. Longitudinal study on thyroid function in patients with thalassemia major: High incidence of central hypothyroidism by 18 years. Indian journal of endocrinology and metabolism. 2013 Nov;17(6):1090.

10. De Sanctis V, Soliman AT, Elsedfy H, Pepe A, Kattamis C, El Kholy M, Yassin M. Diabetes and glucose metabolism in thalassemia major: an update. Expert review of hematology. 2016 Apr 2;9(4):401-8.

11. De Sanctis V, Soliman AT, Candini G, Yassin M, Raiola G, Galati MC, Elalaily R, Elsedfy H, Skordis N, Garofalo P, Anastasi S. Insulin-like growth factor-1 (IGF-1): demographic, clinical and laboratory data in 120 consecutive adult patients with thalassaemia major. Mediterranean journal of hematology and infectious diseases. 2014;6(1). 
12. De Sanctis V, Elsedfy H, Soliman AT, Elhakim IZ, Pepe A, Kattamis C, Soliman NA, Elalaily R, El Kholy M, Yassin M. Acquired hypogonadotropic hypogonadism (AHH) in thalassaemia major patients: an underdiagnosed condition?. Mediterranean journal of hematology and infectious diseases. 2016;8(1).

13. Coşkun E, Keskin A, Süzer T, Sermez Y, Kildaci T, Tahta K. Spinal cord compression secondary to extramedullary hematopoiesis in thalassemia intermedia. Eur Spine J. 1998;7(6):501-504. doi: $10.1007 / \mathrm{s} 005860050114$

\section{FIGURE LEGENDS}

\section{Pathology slides}

1. Figure 1. High magnification image of $\mathrm{H} \& \mathrm{E}$ stained slide showing lymphoid tissue composed of small lymphocytes with scattered megakaryocytes (arrows) and clusters of myeloid and erythroid cells (circled)

2. Figure 2. Immunohistochemical stain "CD61", highlighting the cytoplasm of megakaryocyte in brown chromogen and confirming the lineage.

3. Figure 3. Immunohistochemical stain "MPO", highlighting the myeloid precursors.

4. Figure 4. Immunohistochemical stain "Hemoglobin A", highlighting the erythroid precursors

\section{Radiology images}

1. Sagittal T2 WI of the LSS shows diffuse low signal intensity of the bone marrow of LSS ( known case of thalassemia major) with well-defined mass of the same signal intensity $(5.3 \times 3 \mathrm{~cm})$ in presacral region (blue arrow) and another similar smaller mass $(2 \mathrm{x} 1 \mathrm{~cm})$ in anterior extradural space posterior to S1 level (Blue arrow)

2. Axial post contrast fat saturated image at presacral region shows mild enhancement of the presacral mass (Blue arrow). No definite infiltration of the sacrum.

3. Axial post contrast fat saturated image at S1 level shows mild enhancement of anterior extradural mass (blue arrow) at the midline laterally displacing S1 traversing nerve roots.

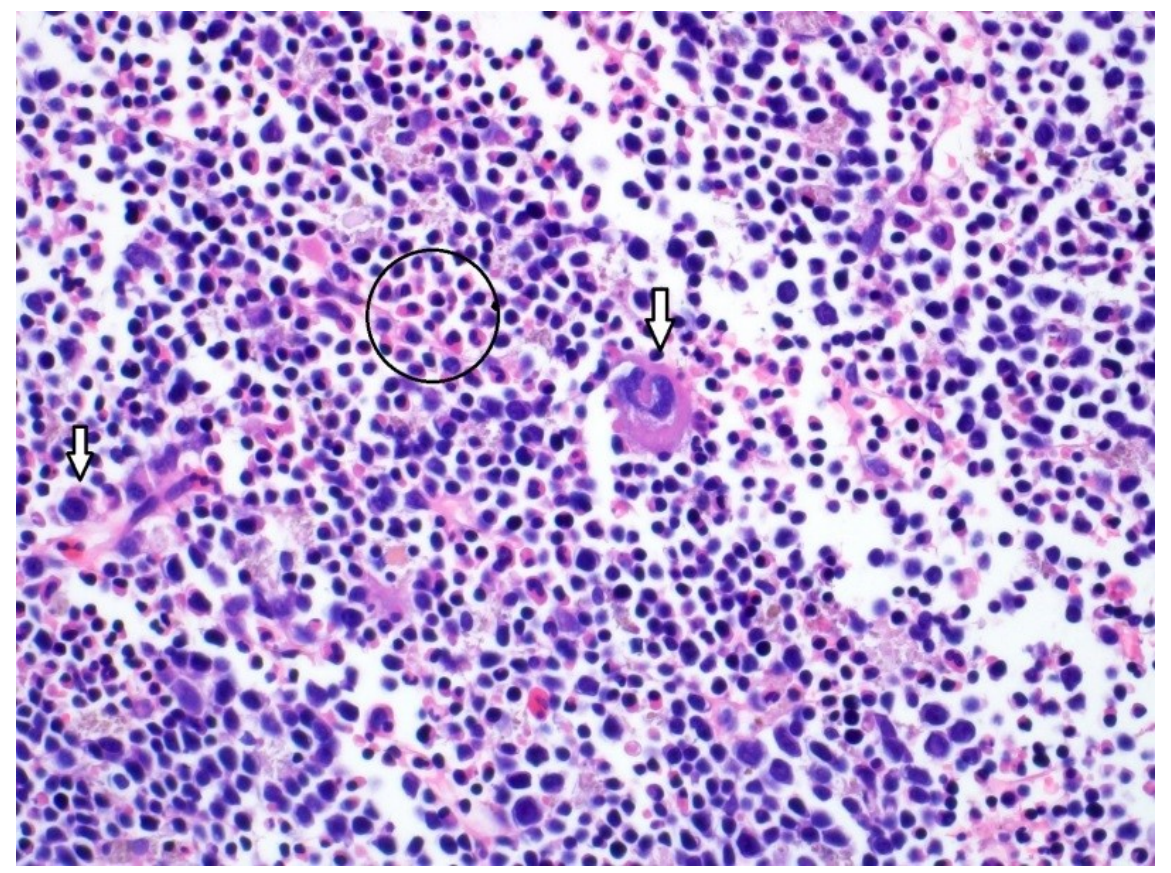



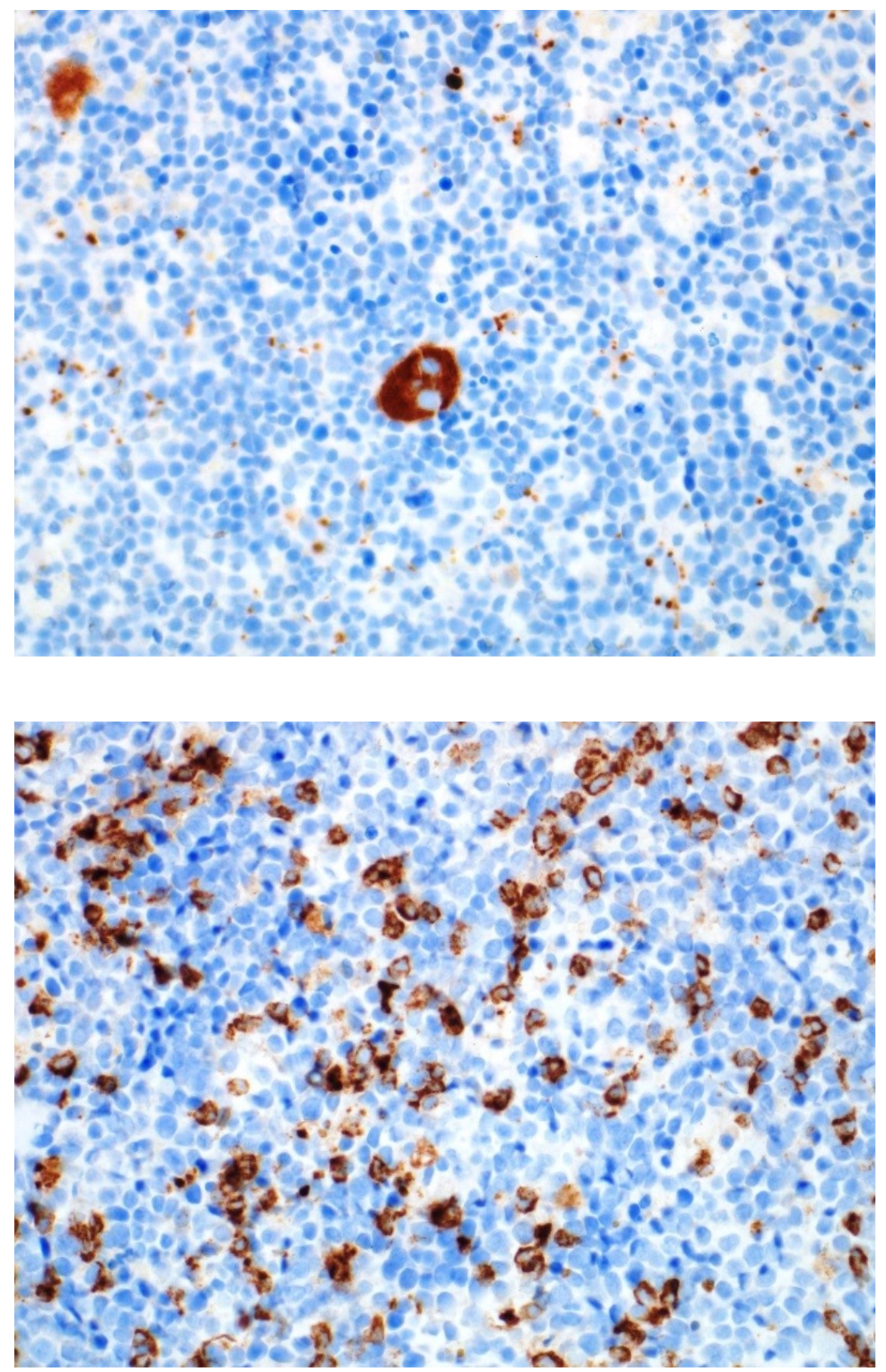

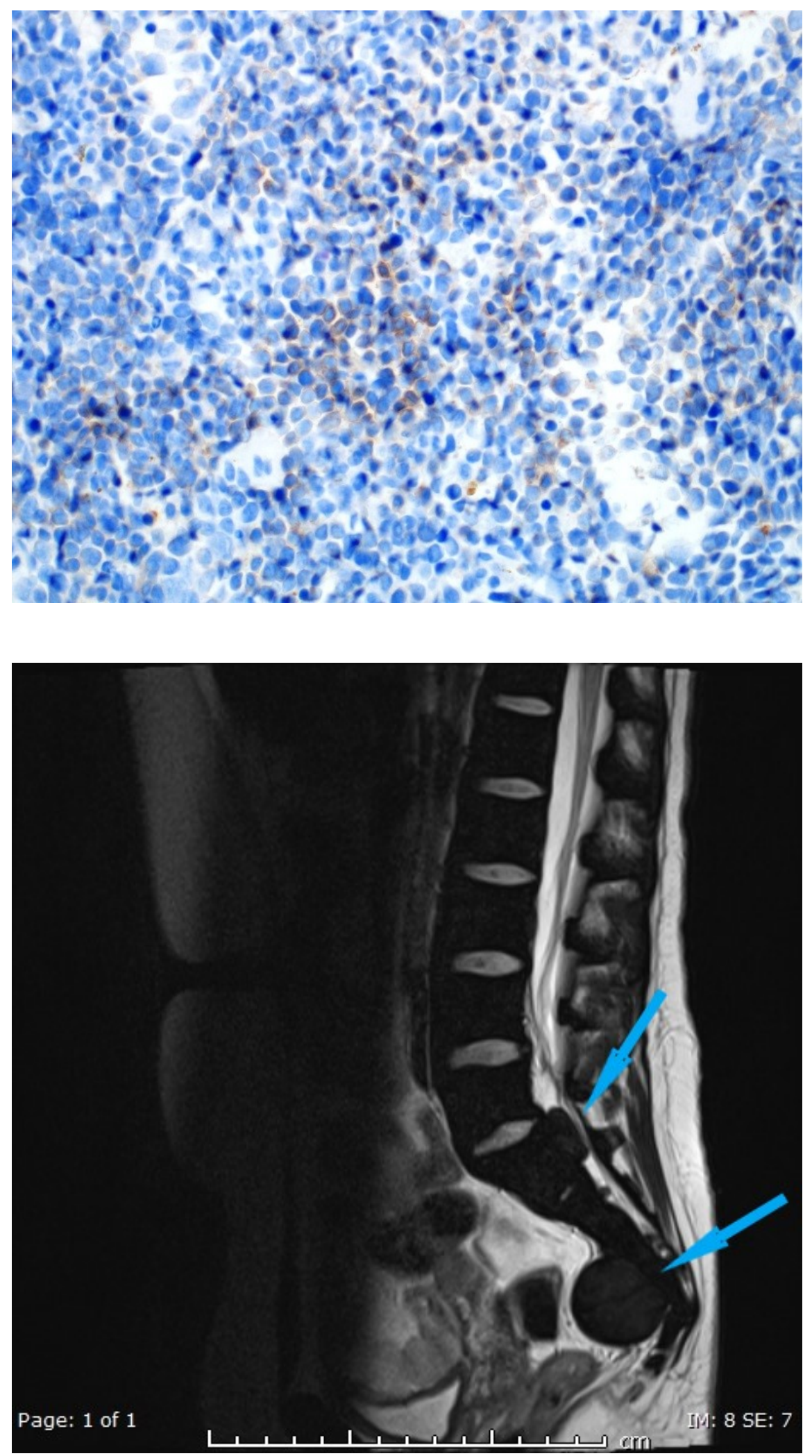


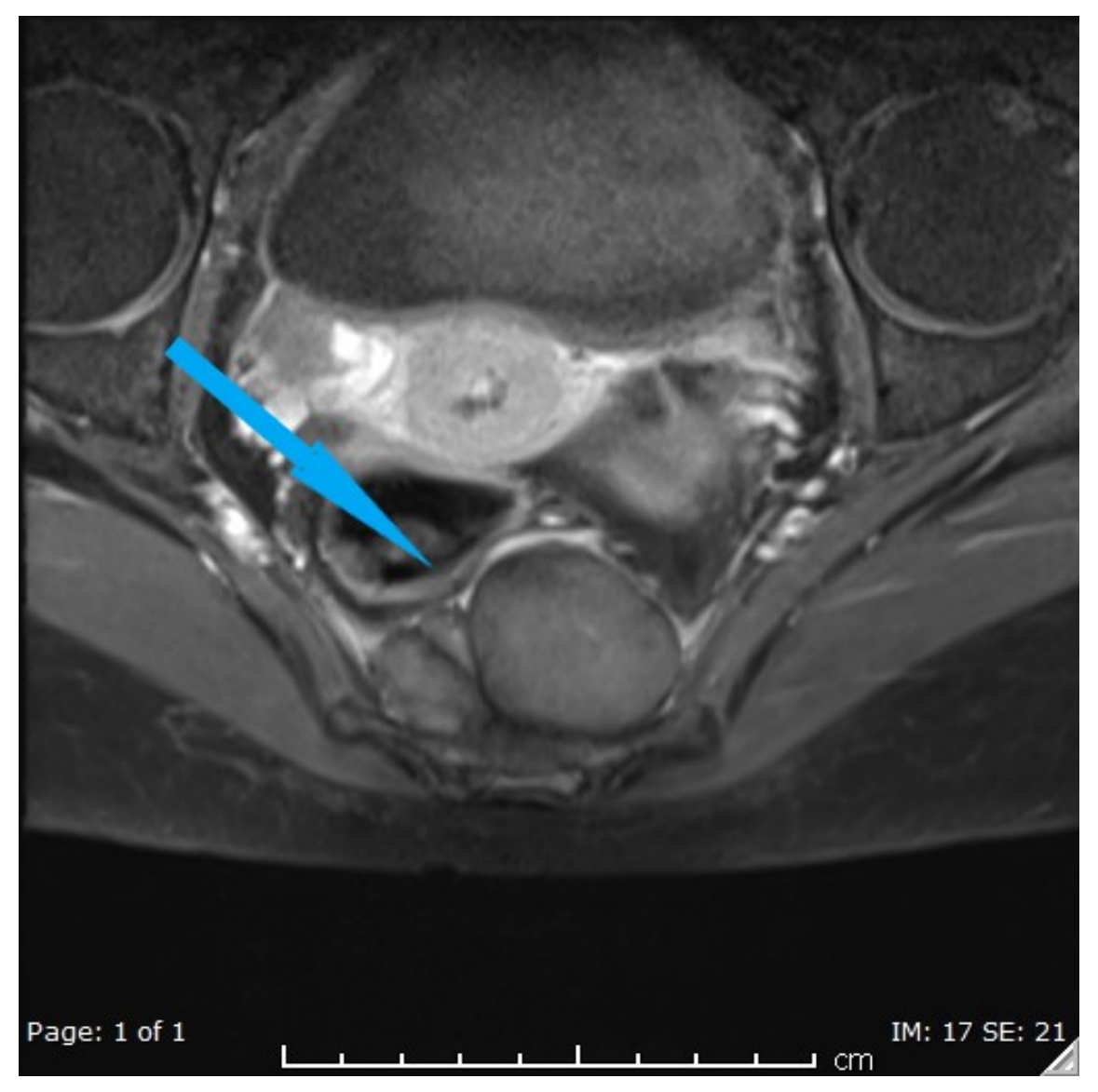




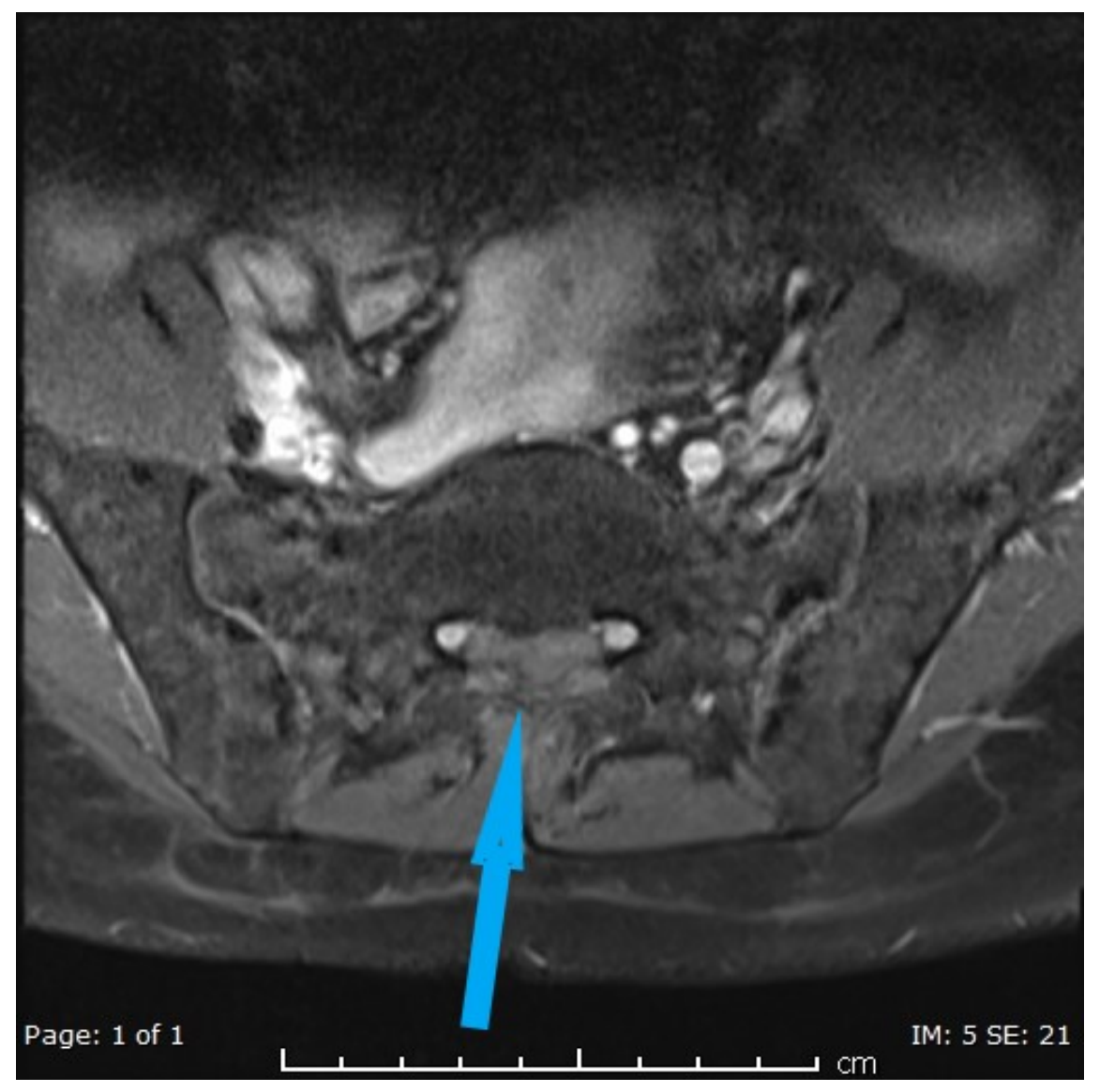

\section{Hosted file}

table.pdf available at https://authorea.com/users/362401/articles/483557-low-back-pain-inbeta-thalassemia-major-revealing-sacral-extra-medullay-hematopoeisis-a-case-report 